\title{
Cambios en la gestión del servicio antimalárico en el estado Sucre
}

\author{
Gómez, Irey* \\ Alarcón, Luis** \\ Velásquez, Ysabel ${ }^{\star \star \star}$
}

\section{Resumen}

Esta investigación intenta dar cuenta de algunos cambios en la gestión del servicio antimalárico en el estado Sucre. En esta revisión se toma como referente empírico, la experiencia del municipio Mariño en el período 2002-2003, siendo este municipio una de las áreas de riesgo malárico existentes en esta entidad federal venezolana. Metodológicamente, se utilizaron técnicas de observación participante, grupos de discusión y entrevistas informales aplicadas a 17 funcionarios de salud adscritos a este servicio. Entre los resultados destacan el proceso de reestructuración institucional que dio lugar a la sustitución de la antigua División de Malariología por la actual Gerencia de Salud Ambiental y Contraloría Sanitaria, así como también la adopción de una estrategia gerencial de carácter participativo, unido al seguimiento y evaluación en forma continua de las estrategias de atención, prevención y control de la malaria. Estos cambios produjeron un impacto positivo en el funcionamiento de este servicio, lo cual se vio reflejado en el control de la endemia durante ese período en las áreas afectadas.

Palabras clave: Descentralización, malaria, gestión social, estado Sucre.

Recibido: 07-06-05 Aceptado: 07-03-06

Trabajadora Social. Docente e Investigadora, adscrita al Departamento de Trabajo Social de la Escuela de Ciencias Sociales de la UDO-Núcleo de Sucre. Coordinadora general del Grupo SOPHIS. E-mail: ireygomez@cantv.net

** Sociólogo-Educador. Investigador y Coordinador de la investigación de Campo en el Grupo SOPHIS. E-mail: gomezalar@hotmail.com

*** Tesista de grado del Departamento de Trabajo Social, Escuela de Ciencias Sociales, Universidad de Oriente. Asistente de Investigación del Grupo de Investigaciones e Innovaciones Sociales - SOPHIS-. 


\section{Changes in Management of the Anti-malarial Service in the State of Sucre}

\section{Abstract}

The purpose of this project was to study some changes in the management of the anti-malarial service in the State of Sucre. For this review, the experience of the Mariño municipality during the period 2002-2003 was taken as an empiric reference point since this municipality is one of the malaria-risk areas in the federal Venezuelan territory. Methodologically, techniques of participative observation were used in discussion groups and informal interviews applied to 17 health functionaries registered with this service. Among the results, those that stood out were the process of institutional re-structuring whereby the former Malariology Division was replaced with the current Management of Environmental Health and Sanitary Control, the adoption of a management strategy with a participative character united with continuous follow-up and evaluation of the strategies for attention to, prevention and control of malaria. These changes produced a positive impact in the functioning of this service, reflected in control of the endemic disease during this period in the affected areas.

Key words: Decentralization, malaria, social management, State of Sucre.

\section{Introducción}

La malaria ${ }^{1}$ es una de las enfermedades más antiguas de la humanidad y en los últimos años se ha convertido en uno de los mayores problemas de salud pública del mundo. Según estimaciones de organismos internacionales de salud (OMS-OPS, 2000) el total de casos clínicos alcanza entre unos 300 y 500 millones anualmente, representando una amenaza no sólo para la salud de los pueblos, sino a su organización social en ge- neral. Se dice que el $90 \%$ de los casos se concentran en África Tropical; sin embargo, otras regiones del mundo como América Latina son seriamente afectadas por esta enfermedad. Específicamente, en nuestro país ha estado presente esta endemia desde épocas pasadas, azotando a gran parte del territorio venezolano. De la situación que existía en el año 1936, caracterizada por el inicio de una amplia campaña, liderizada por el Dr. Arnoldo Gabaldón ${ }^{2}$, que dio como resultado la superación de la malaria como problema de salud pública en los comienzos de la dé-

1 La Malaria es una enfermedad infecto-contagiosa producida por parásitos del genero plasmodium (plasmodium vivax, plasmodium falciparum, plasmodium ovale y plasmodium malariae) que se transmite por los piquetes de la hembra del mosquito anopheles. (Véase: Montes, 2001; Gutiérrez, 1998 y 1999).

2 Arnoldo Gabaldón fue un insigne médico venezolano reconocido por su meritoria labor científica e institucional en la lucha contra la malaria en Venezuela. 
cada de los sesenta. Durante las décadas de los ochenta y noventa, se pasó a otra situación caracterizada por la emergencia y reemergencia ${ }^{3}$ de esta enfermedad, en diferentes zonas del país ${ }^{4}$.

De acuerdo a los organismos regionales de salud, el comportamiento de la malaria en el estado Sucre, revistió un carácter endemo-epidémico ${ }^{5}$ durante el período 1998-2000 ya que se registraron altos Índices Parasitarios Anuales (IPA) ${ }^{6}$ que ubicaron la zona en los primeros lugares como área de riesgo malárico en el territorio nacional. En esta entidad federal, el municipio Mariño ${ }^{7}$ forma parte del área geográfica donde se encuentran los principales focos de infección de la malaria en el ámbito regional.
Dada la importancia que tiene el combate a la malaria en nuestro país, se considera importante la revisión de aquellas experiencias de gestión en salud que dan cuenta de la introducción de innovaciones que han tenido un efecto significativo en cuanto a la disminución de la incidencia de esta enfermedad. El objetivo principal de este trabajo, es describir una experiencia particular de gestión en salud, que está articulada a un proceso político-administrativo de descentralización iniciado en el país a finales de la década de los ochenta, el cual tiene entre sus lineamientos estratégicos el control de algunas enfermedades endemo-epidémicas reemergentes, mediante la reestructuración y racionalización de la organiza-

3 Se conoce como enfermedades emergentes aquellas de tipo infeccioso con incidencia en aumento en las dos últimas décadas. Presentan diversas etiologías, a saber: criónica, viral, bacteriana, micótica o parasitaria, en diversas poblaciones de riesgo con diferentes vías de transmisión histórica- natural y eco-epidemiológica. Por su parte, las enfermedades reemergentes son aquellas de tipo infeccioso conocidas, que reaparecen después de una disminución significativa de su incidencia (Weissenbacher, et. al. 1997).

4 El estado Sucre, junto a los estados Monagas y Delta Amacuro, forman parte del llamado "Foco Oriental" de la malaria en Venezuela, clasificados por la Organización Panamericana de Salud (OPS) como "zonas maláricas de alto riesgo" de transmisión y prioridad de control de esta enfermedad (OPS, s/f). En el caso del estado Sucre, el municipio Mariño-que es el objeto de estudio en este trabajo- se encuentra ubicado en la Península de Paria, cercano al Municipio Cajigal -cuya capital es Yaguaraparo- este último está considerado como uno de los mayores focos de malaria existente el país.

5 Según datos suministrados por la Gerencia de Saneamiento Ambiental y Contraloría SanitariaFundasalud- Sucre, la Malaria fue en ascenso en el estado Sucre. Por ejemplo, en el año 1998 se registraron 4.659 casos de Paludismo; en el año 1999 se presentaron 6.612 casos y en el año 2000 hubo más de 24.500 casos.

6 El Índice Parasitario Anual es la variable básica usada para la estratificación epidemiológica de las zonas de malaria y es la resultante de dividir el número de casos confirmados, entre mil, multiplicado luego por la población en riesgo mediano y alto de presentar la enfermedad. (Organización Panamericana de la Salud -OPS-, 2001).

7 El municipio Mariño limita al norte con el municipio Arismendi, al sur con el Golfo de Paria, al este con el municipio Váldez y al oeste con el municipio Cajigal. Tiene una extensión de 469 km2 y un promedio de 27.107 habitantes (INE, 2001, cit. por Velásquez, 2005). 
ción de este servicio. En el caso de la malaria, este proceso intenta enfrentar, entre otras cuestiones, el modelo de gestión existente hasta ese momento en la llamada División de Malariología, caracterizado por su funcionamiento centralizado basado en una organización jerárquica, integrada por Jefes de Zona, Jefes de División y Jefes de Demarcación. Este modelo tuvo vigencia y se justificaba en un determinado período histórico del país, a partir de la caída de la dictadura gomecista, en el que las condiciones sanitarias y las debilidades en la formación de recurso humano especializado en el servicio antimalárico, requerían de esa estructura centralizada. De hecho a esta unidad administrativa se le atribuye el éxito en la erradicación de la malaria en Venezuela a mediados de los años sesenta. No obstante, a medida que las condiciones políticas, económicas y sociales del país fueron cambiando, el modelo centralizado del sistema de salud, con énfasis en lo curativo, fue debilitándose, cuestión que se expresa en la ineficiencia de las políticas sanitarias. Particularmente, en el servicio antimalárico se traduce en un aparente descuido en el mantenimiento, dotación y/o construcción de infraestructura sanitaria, ineficacia de las actividades de control de vectores, insuficiencias en la formación y asignación de recursos humanos especializados y otras fallas que son atinentes al funcionamiento del sistema de salud en general.

En suma, la experiencia de gestión en salud que referimos en este trabajo, constituye un avance del proceso de descentralización político-administrativo en Venezuela, a pesar de las debilidades que ha presentado su realización en el campo de la salud, especialmente por las diferencias que suelen presentarse entre los aspectos normativos y la realidad. Dicha experiencia nivel local, al menos en el período estudiado, arrojó resultados favorables en relación a la situación sanitaria y específicamente en la disminución de los casos de malaria en esta parte del oriente del país.

Desde el punto de vista teórico-metodológico, se tomaron como referentes los documentos donde se expresa el punto de vista de las organizaciones internacionales de salud (OPS y OMS) acerca de las estrategias para el control de la malaria a nivel mundial. De igual manera, algunos trabajos en relación a la Gerencia Social (Kliskberg, 2003; Molina y Morera, 1999). Pero, principalmente en el abordaje de esta experiencia se intentan poner de relieve las opiniones y vivencias de los actores sociales involucrados en la gestión del servicio antimalárico (gerentes, médicos, enfermeras, inspectores y visitadoras o cazadoras) Personal directivo, Gerencia de Salud Ambiental y Contraloría Sanitaria y personal técnico-administrativo y operativo como: Médicos Jefes de Municipios Sanitarios, Jefes de Demarcación, Epidemiólogos, Enfermeras, Promotores Sociales, Cazadores de Malaria, Visitadores Rurales y Fumigadores). Metodológicamente, se utilizaron técnicas de investigación cualitativa de segundo orden, tales como, la observación participante y las entrevistas no estructuradas. Los informantes clave de este trabajo fueron los funcionarios, calificados como personal directivo y personal técnico-operativo, adscritos a la Gerencia de Salud Ambiental y Contraloría Sanitaria, ubicada en la ciudad de Carúpano, 
estado Sucre. Los entrevistados fueron: Directores, Epidemiólogos, Médicos Jefes de Municipios Sanitarios, Inspectores-Jefes de las distintas Demarcaciones, Enfermeras, Promotores Sociales, Cazadores de Malaria, Visitadores Rurales y Fumigadores.

\section{Gerencia social como escenario de la gestión pública}

En este apartado se presentan en forma breve, algunos puntos relativos a la gerencia social como un referente importante en el desarrollo e interpretación de la experiencia de gestión en salud que se describe en este trabajo.

Así tenemos que la gerencia social intenta superar las concepciones y filosofías tradicionales ${ }^{8}$ en la prestación de los servicios sociales dando paso a nuevas perspectivas y maneras de hacer que proporcionen el mayor bienestar posible a la población. Este enfoque constituye un factor clave para alcanzar una política social eficiente y eficaz. De acuerdo con lo planteado por Kliksberg (s/f), se deben crear las condiciones básicas para que se de una gerencia social que responda a las necesidades reales de la población y supere las limitaciones o "cuellos de boteIla" que entraban la implementación de la política social. Desde su perspectiva, la gerencia social se relaciona con la superación de los déficits sociales de la región y el mejoramiento del funcionamiento de las políticas de inversión en capital humano y capital social, a partir de la optimización del rendimiento de los esfuerzos que lleva a cabo el Estado y otros actores sociales (Kliksberg, 2003).

Esta visión de la gerencia social como una vía que pretende optimizar los recursos comunales e institucionales, es también compartida por Molina y Morera (1999) quienes plantean que en la gerencia social "no hay subordinados sino colaboradores, y la negociación es básica para establecer la red de contactos entre actores". Este es un punto clave ya que la modernización en el ámbito institucional no sólo intenta ofrecer respuestas que orienten la asignación, distribución y ejecución de los recursos sociales hacia un mejoramiento de la calidad de vida sino también la posibilidad de que surja una expresión espiritual y social a través de la ejecución de los programas institucionales.

Lo antes expuesto, parte de un necesario cambio de paradigma que modifique la formas tradicionales de relacionamiento entre el Estado y la sociedad civil. Lo que se plantea hoy desde la gerencia social es un acercamiento de los actores

Las concepciones y filosofías tradicionales de la gerencia se enmarcan dentro de una organización de tipo formal, con un conjunto de normas rígidas e inflexibles, relaciones fundamentalmente verticales, donde la información no fluye, los trámites burocráticos se incrementan, la participación de los actores no es considerada dentro del proceso de toma de decisiones y la evaluación es vista como una amenaza y no como instrumento para el mejoramiento y crecimiento institucional. Bajo esta visión existe también la falsa creencia de que lo público es ineficiente y lo privado no lo es. 
sociales y una valorización de las alianzas estratégicas entre los diferentes actores. La gerencia social precisamente trata de maximizar las sinergias entre los diferentes entes participantes en la gestión de los servicios sociales, con el fin de optimizar los resultados, es decir, se busca la suma de esfuerzos y el desarrollo de la capacidad cooperativa para satisfacer las necesidades e impulsar el desarrollo humano.

No se trata de que uno u otro actor aparezcan como protagonistas del proceso; por el contrario, todos deben tener la misma cuota de participación en la construcción de ese desarrollo. Los actores sociales actúan como socios o cooperadores con y por una meta común: servicios de bienestar social eficientes. De allí precisamente la necesidad de desarrollar alianzas estratégicas en la que todos los actores tienen las oportunidades de aportar ideas para la toma de decisión y las mismas posibilidades en el acceso a la información.

Las tendencias actuales colocan el acento en una gerencia social que tenga una filosofía humanista, donde las relaciones de horizontalidad juegan un papel fundamental en la medida que permiten superar la concepción tradicional de dominación y sumisión, en conjunto con una visión de integración de todos los actores. Desde esta perspectiva el ser humano es visto como un ente creativo que debe ser motivado, con una visión futurista y una formación de carácter general. Por otra parte, el cambio en y desde la gerencia social es visto como una oportunidad que conlleva nuevos retos, nuevas formas de hacer, a diferencia de los modelos tradicionales de gestión donde los cambios y transformaciones son vistos como amenazas para la acción gerencial.

Desde nuestra perspectiva la gerencia social eficiente la constituye aquella que puede generar un impacto positivo en la disminución de la situación de pobreza de la población y potencie sus capacidades de participación activa, consciente y responsable en el desarrollo social. Es decir, un tipo de gerencia que además de dar respuestas a las necesidades sociales de la gente, forme, eduque y capacite para la acción social.

Existe un amplio consenso acerca de que la gestión de las organizaciones y más específicamente las de corte público debe centrarse en un enfoque de gerencia social eficiente, en el cumplimiento de su misión y visión mediante una estructura gerencial flexible y horizontal, que se oriente a la innovación, basada en relaciones interpersonales intra e ínter organizacionales, bajo un clima propicio de confianza y motivación que favorezca el desempeño y la satisfacción laboral. Ello supone, indudablemente, un cambio de paradigma, una nueva concepción de los servicios públicos y un enfoque renovado de gerencia para enfrentar las tareas del Estado como ente encargado de la gestión de los servicios de bienestar social, con el fin de fortalecer en todo momento las bases del poder social de las comunidades; es decir la participación. (Molina y Morera, 1999).

En fin, la gerencia social se presenta como una alternativa u opción de funcionamiento de las instituciones públicas venezolanas, ante las reiteradas críticas que éstas han recibido en relación a un manejo irracional que se muestra incapaz de asumir y enfrentar los retos que impone la búsqueda del desarrollo humano, 
tan necesario en los llamados países en desarrollo.

Al establecer una relación entre la gerencia social, como paradigma orientador de la gerencia de los servicios sociales (Klisberg, 2003) y la experiencia de gestión del servicio antimalárico en el estado Sucre, se detectaron los siguientes elementos:

a) el intento - a veces un tanto intuitivo - de seguir un abordaje de la salud en sentido integral, en sustitución del enfoque sectorial que ha venido predominando en el sistema de salud venezolano.

b) el desarrollo de un estilo gerencial adaptativo, que se impone por las mismas características que presenta el funcionamiento general del sistema de salud y por la manera como se comporta la endemia. Ambas cuestiones suponen una dinámica institucional que debe lidiar con múltiples variables que muchas veces no están en el horizonte de lo normativo. Una gerencia adaptativa consiste en sustituir la tradicional práctica de "gerencia por crisis" por otro estilo que se adapte a las necesidades del entorno de la forma más racional posible.

Por otra parte, encontramos ciertos "vacíos" al comparar la experiencia de gestión en referenciacon lo que teóricamente plantea el paradigma de gerencia social. Pensamos que esto tiene que ver con aspectos que caracterizan actualmente el funcionamiento de los organismos que integran el sistema de salud nacional: la falta de análisis sistemático del medio ambiente, la debilidad o ausencia de redes interinstitucionales y de participación comunitaria -en sentido más amplio- en las distintas fases del proceso de gestión, incluyendo la contraloría social.

\section{Antecedentes en la organización y administración de los servicios antimaláricos en Venezuela: el período de gabaldón}

En este apartado se describen algunos acontecimientos significativos en materia de organización y administración del servicio antimalárico en Venezuela, a partir de la caída de la dictadura de Juan Vicente Gómez, durante el período inicial de modernización del país. En particular se destacan los avances alcanzados en la construcción de un sistema de salud que alcanzó notables resultados en la transformación de las condiciones sanitarias, específicamente en la atención y control de la malaria.

En el año 1936, la malaria adquiere el rango de problema de salud pública y se convierte en una prioridad y responsabilidad del Estado venezolano cuando el Congreso Nacional sanciona la Ley de Defensa Contra el Paludismo. La aprobación de esta Ley permitió la creación de la División Especial de Malariología, la cual venía funcionando como una dirección especial del para ese entonces Ministerio de Sanidad y Asistencia Social (MSAS) ${ }^{9}$. El primer director de esta 
División fue el Doctor Arnoldo Gabaldón, es un hecho conocido que este médico venezolano emprendió una obra significativa en la lucha contra esta endemia que incidió a su vez en el mejoramiento de las condiciones sanitarias del país en aquel momento.

De acuerdo a Gutiérrez (1998; 1999) la División de Malariología al iniciarse la gestión del Dr. Gabaldón se caracterizaba por la inexistencia de una organización estructurada capaz de dirigir la lucha antipalúdica. Para superar esta limitación se tomaron como referencia modelos organizativos provenientes de otros países, los cuales fueron adaptados a la realidad venezolana. Por un lado, se establecieron pautas claras en los manuales de funciones sobre el quehacer de cada funcionario (esto debido a la falta de instrucción educativa de gran parte del personal), y se controlaba muy de cerca la ejecución del trabajo por medio de las supervisiones. Por el otro, se motivaron y apoyaron las iniciativas derivadas de la experiencia que se iba adquiriendo y se amplió la formación del personal a través de cursos de capacitación.

Un aspecto resaltante de este período, es la existencia de una Escuela de Expertos Malariólogos, así como también de un Laboratorio de Control donde se revisaba el material clasificado en el campo, de una oficina para la revisión de las labores epidemiológicas y un servicio de contabilidad y depósito. Sin embargo, desde el principio Gabaldón propuso la necesidad de trasladar hasta las mismas localidades, parte importante de las actividades que realizaba la División. Fue así, como se organizó toda una red de estaciones experimentales dotadas de per- sonal y equipo de laboratorio para procesar el material recolectado en las comunidades, realizar los estudios necesarios y planificar la ejecución de los programas de control. Estas estaciones tenían asignado un personal profesional integrado por médicos e ingenieros, otras estaban bajo la responsabilidad de inspectores entrenados para adelantar bajo supervisión, aspectos básicos, tales como el control de vectores.

Las estaciones, a su vez, eran organizadas en tres secciones: la de Epidemiología encargada de los estudios parasitológicos, entomológicos y de quinización. La sección de Ingeniería con el apoyo de uno o más topógrafos y obreros responsables de planificar y construir las obras de ingeniería antimalárica. Y la sección de Administración encargada de la compra de equipos y materiales y del manejo del personal. Con el transcurrir del tiempo la División de Malariología creció y se diversificó. Esto dio origen a la división en Zonas Administrativas dirigidas por un profesional (médico y/o ingeniero) responsable de la marcha del trabajo y vínculo formal entre la Zona y el Jefe de División. La organización burocrática fue acompañada con la creación de instancias no formales que apoyaron el trabajo de la División, tales como: las "Comisiones Locales de Lucha Antimalárica”, las cuales fueron establecidas bajo la iniciativa de los municipios para realizar labores antilarvarias con el apoyo financiero de las rentas de los municipios o de los gobiernos estadales. También, esta organización se encargó de divulgar ante la comunidad los beneficios de las actividades antimaláricas. Igualmente, se crearon las "Brigadas Antipalúdicas" impulsadas por 
personas de las propias comunidades para motivar a la colectividad en su problema malárico y obtener apoyo para las acciones de la División (Gutiérrez, 1998).

En este período, la División de Malariología tenía una dirección centralizada con pautas generales que emanaban de la oficina central, esto se justificaba debido a la falta de personal y a la escasa experiencia del que ya existía, lo cual exigía la supervisión constante del trabajo que realizaban estas personas. La División tenía una estructura jerárquica piramidal en cuya cúspide se encontraba Gabaldón quien concentraba la autoridad y definía las líneas generales de trabajo, regulando en detalle cada una de las acciones a realizar por la dependencia. La División funcionaba en varios circuitos. En el primer circuito, se encontraban los inspectores y el personal de campo. Los inspectores debían supervisar el trabajo del personal de campo, sintetizar su trabajo y responder ante su inmediato superior. El segundo circuito, estaba integrado por los inspectores y los profesionales encargados de supervisarlos. Cada inspector debía presentar un informe a su supervisor quien lo analizaba y si encontraba alguna discrepancia, verificaba la información visitando los criaderos y las viviendas de las localidades. Por su parte, a los médicos les correspondía evaluar clínicamente a los que presentaban fiebre, además de medir junto al inspector más experto los bazos de los escolares y de las personas de las localidades y llevar también un diario donde recogían las impresiones de interés sociológico, epidemiológico y administrativo. El tercer circuito, lo integraban los profesionales de mayor jerarquía (Jefes de Zona) y el Di- rector. Los Jefes de Zona entregaban cuenta semanal y/o mensual al Director, dependiendo de la distancia y de la urgencia de los problemas sanitarios en relación a la malaria.

Otro aspecto interesante de este período, es que la División de Malariología instituyó reuniones mensuales donde participaba fundamentalmente el personal profesional. En estas reuniones se discutían los avances de los estudios e investigaciones, los obstáculos encontrados y los resultados, así como también los métodos publicados por investigadores de otros países que servían para explicar la situación de la malaria a nivel local.

En fin, el funcionamiento de la División en referencia giraba en torno a sus instancias burocráticas (Jefes de Zona, Jefes de División y Jefes de Demarcación). Un elemento que también vale la pena mencionar, es el valor que tuvo para la gestión la relación afectiva creada entre Gabaldón y el personal de la División, lo cual a juicio de Gutiérrez (1998) generó un sistema de lealtades que favoreció el desarrollo de la institución y el control de la Malaria en los inicios de la década de los sesenta. Se tipificó un liderazgo paternalista por parte del Dr. Gabaldón, aunado al funcionamiento centralizado y vertical del servicio antimalárico durante este período. Aún así, en la historia de Venezuela no queda lugar a dudas acerca del papel decisivo que jugó la División de Malariología durante la gestión del Dr. Arnoldo Gabaldón, en la erradicación de la malaria como problema de salud pública a nivel nacional. La estrategia gerencial implementada por este médico permitió que 
para el año 1963 se declarara erradicada esta enfermedad del territorio venezolano ${ }^{10}$.

\section{La gestión del servicio antimalárico en los años ochenta y noventa}

Desde la década de los ochenta, a partir de la crisis del modelo económico en Venezuela, el sistema de salud se ve seriamente afectado. Entre otras cosas, esto se refleja en el descuido de las políticas sociales y especialmente de las políticas sanitarias antimaláricas, aunado al deterioro de la calidad de vida de la población. En los años ochenta se produce la emergencia y reemergencia de la malaria después de un prolongado receso o disminución de los índices de casos relativos a la misma. En un breve recuento de esta situación se tiene que para el año 1988, a nivel nacional se registró un promedio de 42.662 casos, manteniéndose la prevalencia de esta enfermedad con cifras similares hasta el año 1991. Posteriormente, en el año 1992 se detectaron 21.417 casos y para el año 1993 disminuyó a 8.145 casos, aunque la presencia de esta enfermedad seguía representando un problema de salud pública (Universidad de Cara- bobo, 2000). En el año 1998 se registra en el territorio nacional un total de 21.682 casos (OPS/OMS, 2000b), siendo los estados Bolívar y Sucre, las zonas de mayor riesgo palúdico. Una cifra bastante cercana ( 21.585 casos) se reporta para el año 1999 , mientras que para el año 2000, específicamente en la semana 21 se registraron 16.277 casos. Es importante acotar que este panorama sanitario se produce en un contexto caracterizado por la crisis económica nacional de los años ochenta, seguida durante la década de los años noventa de la aplicación de políticas económicas de ajuste, las cuales en el campo de la salud influyeron entre otras cuestiones, en el reforzamiento del esquema curativo en salud en detrimento de la inversión en el área preventiva y en la disminución del gasto público. Adicionalmente, los intentos de modernización del sistema de salud tuvieron escaso impacto en el funcionamiento eficiente del servicio antimalárico.

En este escenario, el estado Sucre ha sido una de las entidades mayormente afectadas por la presencia de la malaria. En la década de los noventa, aunque con leves variaciones, hubo una marcada incidencia de esta enfermedad, siendo mayor al final de la década ${ }^{11}$, al punto que esta entidad federal fuese declarada

10 Entre los resultados se destacan para el año 1961, Venezuela logró erradicar el paludismo o malaria en un área de 430.000 km2 y para 1968, esta cifra se elevó a 460.000 km2, situación que también está asociada a un mejoramiento progresivo de las condiciones socio-sanitarias del país.

11 En el año 1990 se produjeron 6.842 casos; en el año 1991 aumentó a 8.231 casos; en el año 1995 descendió a 3.777 casos; en el año 1996 se mantuvo en 4.763 casos; en 1997 aumentó a 6.964 casos; en el año 1998 se mantuvo en 6.612 casos; en el año 1999 disminuyó a 4.659 casos y finalmente en el año 2000, hubo un ascenso significativo a $11 . .376$ casos (Velásquez, 2005). 
como una zona de riesgo ya que en varias ocasiones se presentaron situaciones de epidemia. Cabe destacar que la reemergencia de la malaria no produjo inmediatamente la revisión de la estrategia antimalárica que se venía aplicando hasta ese momento. Se continuó con la misma estructura que había fundado Gabaldón, es decir, la lucha antimalárica era responsabilidad de la División de Malariología. Aunque esta División había experimentado cambios con relación a la expansión de su estructura y su personal, estos cambios no resultaban efectivos frente al acelerado crecimiento de la población y de los focos de infección que surgían en las zonas afectadas. De tal manera que se llega al año 2000 y dada la situación en cuanto a la incidencia de la malaria, existen elementos para pensar que se había producido un colapso de la estrategia antimalárica existente en la región en ese momento. De acuerdo a los registros epidemiológicos de los organismos competentes, en el caso del Municipio Mariño, durante el período 1999-2002 -a excepción del año 2001- es visible el aumento de los Índices Parasitarios Anuales (IPA) lo cual representa el principal indicador de un aumento de la enfermedad en esa zona. En el año 1999, el número de casos fue de 883 y el IPA de 40,6; para el año 2000 , esta cifra ascendió a 1.134 casos, con un IPA de 52,1; para el año 2001 descendió notablemente, registrándose una cifra de tan sólo 227 casos y un IPA de 9,6; llegando en la semana epidemiológica № 39 del año 2002 a un ascenso de 1.230 casos y un IPA de 92,9 (Gerencia de Salud Ambiental y Contraloría Sanitaria, 2002).

Sin embargo, como toda crisis representa siempre una oportunidad, este panorama negativo que se presentó con respecto a la incidencia de la malaria ha sido revertido en los últimos años. En el caso del municipio Mariño -que hemos tomado como referencia para indicar el éxito en la disminución de esta endemia, tenemos que según los registros epidemiológicos de los organismos regionales de salud, para el año 2003 sólo se registraron 263 casos en el municipio antes referido. Este registro indica una merma significativa de la malaria en comparación con lo registrado en los años anteriores y como se verá más adelante, según lo expresado por el personal de salud, es el resultado de la aplicación de nuevas estrategias gerenciales en el combate a esta enfermedad.

A finales del año 2001 e inicios del año 2002, en el marco de la descentralización de la salud en el estado Sucre y de los planteamientos hechos por la Organización Mundial de la Salud (OMS) y la Organización Panamericana de la Salud (OPS) en el documento que recoge la "Estrategia Global para el Control de la Malaria" (2001) se empieza a generar un proceso de reestructuración de la anterior División de Malariología, el cual incluyó una serie de cambios en cuanto a la gerencia de este servicio en la atención, prevención y control de dicha patología. Esta experiencia es la que en el siguiente apartado intentamos recoger a partir principalmente de los testimonios de una parte del personal de salud. 


\section{La reestructuración de la división de malariología en el estado Sucre}

El proceso de reestructuración tiene relación con la descentralización del sistema de salud a nivel nacional y con el cumplimiento de pautas establecidas a nivel internacional en la atención de la malaria, dictada por la OMS y OPS. Esto último contempla una estrategia de abordaje integral del problema de la malaria, mediante un esfuerzo coordinado entre el servicio antimalárico, el personal de salud perteneciente a los Municipios Sanitarios, los habitantes de las comunidades ubicadas en las zonas de riesgo y otros organismos públicos y privados. Es decir, el reconocimiento de la malaria como un problema de salud pública cuya atención requiere la participación activa de distintos actores vinculados a este problema.

En el caso del estado Sucre, se produjo la adscripción de la antigua División de Malariología al principal organismo responsable de la salud, como lo es la Fundación para la Salud del Estado Sucre (FUNDASALUD). Valga decir que FUNDASALUD se crea en el marco de la descentralización de este servicio en la región sucrense. ${ }^{12}$ Con esta reestructuración esta División pasó a formar parte de la estructura de dicha Fundación bajo el nombre de Gerencia de Salud Ambiental y Contraloría Sanitaria. También se integraron a esta Gerencia, los llamados Municipios Sanitarios (antiguos Distritos Sanitarios) y con ello la prestación de los servicios asistenciales de salud (incorporación de médicos, enfermeras y personal de promoción social que anteriormente no estaban directamente involucrados en la prestación del servicio antimalárico). Con esta fusión, el abordaje de la malaria se centra en los siguientes ejes: actividades médicas; educación para la salud; control vectorial e investigación. Específicamente, lo que antiguamente se conocía como Malariología se dedica principalmente a las actividades de control de vectores e investigación epidemiológica y entomológica, mientras que los Municipios Sanitarios cumplen actividades médico-curativas y de prevención; entre ellas, la detección, diagnóstico, tratamiento e investigación epidemiológica de la enfermedad. Lo que en el lenguaje que maneja el personal de salud, se conoce como el nuevo "DDT" (Detección, Diagnóstico y Tratamiento $)^{13}$.

12 En el marco de los convenios de transferencia de competencias del sistema de salud en Venezuela, se creó FUNDASALUD según el decreto № 033 el 24 de junio de 1993, publicado en Gaceta Oficial Extraordinaria № 89 del 19 de julio de 1993. Este organismo está adscrito a la Gobernación del estado Sucre, tiene personalidad juirídica propia y es el máximo responsable del sistema de salud en esa entidad federal. En este marco también se promulga la Ley de Salud del estado Sucre, publicada en Gaceta Oficial № 234 de fecha 20 de febrero de 1996, en esta Ley se describen las acciones legales para el funcionamiento del servicio descentralizado de salud del estado Sucre (Cumana Y y Marchán Y, 2005).

13 EI DDT de los tiempos del Dr. Gabaldón era un producto químico de nombre Diclorodifenil-Tricloroetano cuya finalidad era actuar sobre los vectores e interrumpir su período de vida. De ahí 
Otro aspecto importante en esta nueva forma de gestión es la planificación en forma participativa y de acuerdo al seguimiento continuo del comportamiento de la endemia. Es decir, se elaboran planes de trabajo en función de la información epidemiológica que aportan los Municipios Sanitarios y se realizan de manera conjunta las actividades de educación para la salud, es decir las acciones preventivas; evaluaciones epidemiológicas y entomológicas. Progresivamente, se ha ido incorporando a la comunidad, mediante distintas vías, a saber: la búsqueda pasiva (los pacientes buscan atención médica ante cualquier síntoma); la búsqueda activa (el personal de salud detecta los casos o pacientes); la participación de habitantes de la comunidad como voluntariado en labores de vigilancia epidemiológica (es el caso de las Ilamadas "cazadoras de malaria").

Cabe destacar que la Gerencia de Salud Ambiental y Contraloría Sanitaria conserva la estructura organizativa de tipo piramidal de la antigua Malariología pero se diferencia de ella en cuanto el ac- tual equipo gerencial ha adoptado como norma mantener con todo el personal relaciones de tipo horizontal, en la que todos tienen la oportunidad de expresarse abiertamente, y donde los funcionarios participan en el proceso de toma de decisiones, cuestión que expresa una gestión de carácter participativo ${ }^{14}$. De igual manera, se mantiene una comunicación constante en el equipo de salud, fomentándose además un espíritu de trabajo que lógicamente favorece el desarrollo del servicio. El Cuadro 1 recoge sintéticamente los aspectos considerados más significativos del "antes" y "después" del proceso de reestructuración.

\subsection{Algunos elementos que caracterizan la gestión del servicio antimalárico después de la reestructuración}

Indudablemente que el análisis de los elementos referidos en el Cuadro antes presentado requiere un espacio mayor que el dedicado en el presente trabajo, a fin de que esta experiencia pueda servir de referencia en otros ámbitos que

que en este proceso de reestructuración se tomaron las siglas de este producto que marcó una etapa en la lucha contra la malaria pero con un nuevo significado. En este contexto estas siglas -en el imaginario del personal de salud- ya no refieren a un producto químico sino a lo que se conoce como "Detección, Diagnóstico y Tratamiento" en forma oportuna e inmediata, destacando así una visión que va más allá del tradicional control de vectores, dando importancia a la prevención mediante la participación directa de la comunidad y la actuación inmediata del personal de salud.

14 Esto se expresa en las reuniones técnico-administrativas que se realizan en forma semanal con todo el personal que integra esta Gerencia. Dichas reuniones constituyen un ámbito de trabajo donde se monitorea la situación epidemiológica en los municipios, se analiza la situación, se proponen, discuten y deciden planes de trabajo que en síntesis responden a la estrategia de abordaje de la malaria en la región. Valga destacar que en estas reuniones también suelen participar, representantes del poder público local, organismos educativos e investigadores universitarios. 


\section{Cuadro 1 \\ Síntesis de los cambios generados a partir de la reestructuración}

Aspectos que definen la gestión
Tipo de personal responsable del
control, tratamiento, prevención y
atención de la Malaria.

Racionalidad predominante

Investigación de la enfermedad

Tipo de liderazgo

que se promueve.

Percepción de la Malaria

\section{Antes}

Después

División de Malariologìa

Única y exclusivamente el personal de la división de Malariologìa. Visitadores rurales, pastilleros, jefes de Zona, Jefes de demarcación, entre otros.

Racionalidad curativa fundamentada en la distribución masiva de drogas antimalàricas (quinizaciòn) y control de vectores que consiste en el rociamiento del interior de las viviendas y sus alrededores con DDT.

Centrada en factores epidemiológicos, entomológicos y físico ambientales.

Basado en relaciones fundamentalmente verticales, de arriba hacia abajo

Es erradicable con distribución masiva de drogas antimalàricas y control vectorial
Dirección de Saneamiento Ambiental y Contraloría Sanitaria

Personal dedicado al control de vectores, adscritos a la Gerencia de Saneamiento Ambiental en conjunto con el personal de salud adscritos a los Municipios Sanitarios (médicos, epidemiólogos, enfermeras, promotores sociales, cazadores de Malaria) Personal que trabaja en equipo en forma coordinada en la atención de la malaria.

Racionalidad preventiva fundamentada en el nuevo DDT (Detección, Diagnóstico temprano y Tratamiento inmediato. Se privilegia las actividades de educación para la salud y participación comunitaria en la prevención de enfermedades y promoción de la salud.

Centrada en factores epidemiológicos, entomológicos, físico-ambientales, socioculturales y psicosociològicos.

Basado en relaciones de horizontalidad, de abajo hacia arriba.

Es controlable por medio de la prevención y participación comunitaria además de las otras actividades. 


\section{Cuadro 1 \\ Continuación}

\begin{tabular}{|c|c|c|}
\hline & Antes & Después \\
\hline Participación de otros actores & Unisectorial & $\begin{array}{l}\text { Intersectorialidad. Fortaleci- } \\
\text { miento de Redes. Todos los } \\
\text { actores sociales. }\end{array}$ \\
\hline $\begin{array}{l}\text { Estrategia de atención utilizada para } \\
\text { detectar la malaria }\end{array}$ & $\begin{array}{l}\text { Búsqueda activa de ca- } \\
\text { sos por el personal de } \\
\text { salud en las comunida- } \\
\text { des. }\end{array}$ & $\begin{array}{l}\text { Hay una tendencia a fortale- } \\
\text { cer la llamada Búsqueda pa- } \\
\text { siva de casos. }\end{array}$ \\
\hline Participación comunitaria & $\begin{array}{l}\text { Los visitadores rurales } \\
\text { y/o pastilleros es un per- } \\
\text { sonal de la División de } \\
\text { Malariologìa que recibe } \\
\text { la debida formación y } \\
\text { luego es asignado para } \\
\text { trabajar en cualquier co- } \\
\text { munidad con problemas } \\
\text { de transmisión malàrica. }\end{array}$ & $\begin{array}{l}\text { Los cazadores de malaria } \\
\text { forman parte del personal } \\
\text { voluntario de la Gerencia de } \\
\text { Salud Ambiental que luego } \\
\text { de recibir la debida capacita- } \\
\text { ción realiza en su propia co- } \\
\text { munidad las labores de de- } \\
\text { tección, diagnóstico y trata- } \\
\text { miento de malaria. }\end{array}$ \\
\hline $\begin{array}{l}\text { Actitud del personal médico no } \\
\text { adscrito a Malariología en cuanto a } \\
\text { la participación comunitaria }\end{array}$ & $\begin{array}{l}\text { Escasa o nula promo- } \\
\text { ción de la I participación } \\
\text { comunitaria en la aten- } \\
\text { ción del problema de la } \\
\text { malaria. Énfasis en los } \\
\text { procesos curativos. }\end{array}$ & $\begin{array}{l}\text { Se busca la participación co- } \\
\text { munitaria en la prevención, } \\
\text { atención y control de la ma- } \\
\text { laria por medio de la forma- } \\
\text { ción de grupos de salud y sa- } \\
\text { neamiento ambiental y la } \\
\text { realización de labores de } \\
\text { control vectorial con la debi- } \\
\text { da capacitación y supervi- } \\
\text { sión del personal de salud. }\end{array}$ \\
\hline Toma de decisiones & $\begin{array}{l}\text { Es débil. Por lo general, } \\
\text { hay actores que desco- } \\
\text { nocen el problema de } \\
\text { malaria y los mecanis- } \\
\text { mos de atención, trata- } \\
\text { miento y prevención de } \\
\text { esta enfermedad. }\end{array}$ & $\begin{array}{l}\text { Presente. Involucrado den- } \\
\text { tro del equipo de salud anti- } \\
\text { malárico. Se discute y se co- } \\
\text { noce con mayor amplitud el } \\
\text { problema y las medidas de } \\
\text { atención y a través del per- } \\
\text { sonal médico y de promo- } \\
\text { ción social se relaciona con } \\
\text { las comunidades afectadas. }\end{array}$ \\
\hline
\end{tabular}




\section{Cuadro 1 Continuación}

\begin{tabular}{|c|c|c|}
\hline & Antes & Después \\
\hline $\begin{array}{l}\text { Evaluación y monitoreo } \\
\text { del trabajo realizado }\end{array}$ & $\begin{array}{l}\text { Supervisada. Unilateral. } \\
\text { Controlada. }\end{array}$ & $\begin{array}{l}\text { Libre en base a criterios y } \\
\text { análisis de la situación pre- } \\
\text { sentada. Por consenso. Se } \\
\text { sistematiza la información } \\
\text { mediante la presentación de } \\
\text { informes y la automatización } \\
\text { de la "data" o casuística de } \\
\text { malaria en forma semanal. }\end{array}$ \\
\hline Reuniones & $\begin{array}{l}\text { Por informes y reunio- } \\
\text { nes con el supervisor in- } \\
\text { mediato. }\end{array}$ & $\begin{array}{l}\text { Por informes. Reuniones de } \\
\text { equipo de trabajo (reuniones } \\
\text { con personal técnico opera- } \\
\text { tivo en el ámbito municipal, } \\
\text { reuniones técnico adminis- } \\
\text { trativas, con el personal de } \\
\text { todo el estado, y reuniones } \\
\text { interestadales con personal } \\
\text { de los diferentes estados del } \\
\text { país con problemas de } \\
\text { transmisión malàrica) Visi- } \\
\text { tas de las áreas de trabajo. } \\
\text { Inspecciones presénciales. }\end{array}$ \\
\hline
\end{tabular}

Fuente: Elaboración propia.

sufren el problema de la malaria. Por tratarse de una investigación en curso, nos centramos sólo en la descripción de algunas variables, tales como la participación comunitaria: en forma de voluntariado a través de la figura del "cazador o cazadora de malaria" y de otros actores bajo el concepto de "intersectorialidad" y las opiniones de algunos funcionarios entrevistados (médicos, inspectores sanitarios, promotores sociales) en relación al proceso de reestructuración del servicio antimalárico. El proceso de investigación se basó en entrevistas no estructuradas y observación participante aplicadas por los investigadores mediante su asisten- cia a las reuniones técnico-administrativas realizadas por el personal de la Gerencia en referencia y a las actividades médico-sanitarias en el área de estudio.

El proceso de reestructuración permitió la incorporación de la figura del "Cazador" o la "Cazadora" de malaria. Se trata de un personal voluntario, en su mayoría del sexo femenino. En parte, vienen a sustituir a los anteriormente llamados "pastilleros" y/o visitadores rurales de los tiempos de Gabaldón. Su trabajo es altamente apreciado ya que en su selección se toma en cuenta la pertenencia a la comunidad donde prestan sus servicios, como estrategia de los organismos de sa- 
lud para garantizar la detección, seguimiento y asistencia oportuna a los enfermos de malaria.

La figura del cazador ha sido una pieza importante en los logros alcanzados en la disminución de la enfermedad. El hecho de tener su residencia y de realizar su trabajo en las zonas de riesgo malárico obviamente le otorga ciertas ventajas en la promoción de la participación comunitaria, que no tiene el personal médico-asistencial que atiende desde los centros de salud. A la "cazadora" se le facilita el trabajo comunitario, el contacto y la inserción en los lugares donde viven los pacientes ya que conoce la zona y en cierto modo comparte las pautas culturales de la población, porque forma parte de ella y conoce sus costumbres. De ahí que casi siempre los pacientes y familiares le abren con mayor confianza las puertas de los hogares, al momento de hacer las visitas para administrar tratamiento o llevar a cabo la vigilancia epidemiológica. Además, los cazadores permanecen la mayor parte del tiempo en la comunidad y ante el menor síntoma suelen ser solicitados por los afectados antes de acudir a los centros de salud. Parte de su trabajo es identificar en forma rápida, la presencia de la enfermedad mediante la realización del examen respectivo para llegar al diagnóstico. La diferencia con el período anterior es que el pastillero tomaba las muestras de los "casos sospechosos" y se marchaba; si éstos resultaban positivos eso se conocía pasadas las 72 horas. Este modo de operar aumentaba las posibilidades de transmisión entre los que habitaban en un mismo hogar o vecindario.
Hoy día, con la ayuda del cazador, las actividades de detección, diagnóstico e incluso tratamiento han sido trasladadas a la misma comunidad. De modo que la Detección Temprana es un hecho. Otro cambio es en relación al suministro del tratamiento de malaria en la propia casa del paciente. Esto ya se venía ejecutando pero ahora el medicamento es entregado a los pacientes con la obligación de ingerirlo en presencia del personal voluntario o cazador. De ese modo se garantiza la continuidad y culminación efectiva del tratamiento. Este tipo de ayuda es de gran utilidad para los médicos, enfermeras y el resto del personal de salud, quienes tratan a través de la cazadora, de activar las llamadas "Búsqueda activa" y "Búsqueda pasiva" en el control de la malaria.

Pro otra parte, la percepción que tiene el personal de salud acerca de la cazadora, es que su trabajo garantiza el cumplimiento del tratamiento contra la malaria. Al respecto, un funcionario entrevistado lo expresó del siguiente modo:

-. "(...) anteriormente cuando no existían ellos refiere a los cazadores pues los visitadores dejaban el tratamiento, no cubrían toda la población y había que darles tratamiento de dos a tres días, (...); se hizo un muestreo en esa zona y se encontraron todavía en algunas viviendas, los medicamentos envueltos en el mismo papel que se lo dejaban, a raíz de allí, empezaron a trabajar los cazadores, y se les prohibió terminantemente dejar el trata- 
miento al paciente". (06- 07- 16) ${ }^{16}$ (Médico).

Es oportuno aclarar que la incorporación del cazador de malaria forma parte de una estrategia gerencial que en cierto modo viene a cubrir la deficiencia de personal existente en la antigua Malariología, reforzando el trabajo en los tiempos de alta incidencia de la enfermedad. Sin embargo, la insuficiencia presupuestaria que existe en este organismo actúa en contra de las propias cazadoras ya que el bono que reciben por su trabajo, por lo general no es cancelado oportunamente, cuestión que obviamente no es coherente con la responsabilidad que le es asignada, representando también un motivo de preocupación ya que por lo general las condiciones socio-económicas de ella y su grupo familiar son precarias. La mayor parte de las cazadoras tienen la expectativa de conseguir un empleo fijo o eventual que le permita mejorar sus ingresos, por eso procura siempre proyectar una imagen positiva de su trabajo y se entrega al mismo de manera extraordinaria, a sabiendas de que vive en una zona donde las fuentes de empleos son limitadas y la cantidad de desempleados es muy alta. ${ }^{17}$ Esto situación forma parte del lado oscuro del trabajo voluntario ya que en condiciones de desempleo y bajos ingresos del grupo familiar, no existe compensación, no tanto hacia el trabajo voluntario sino en forma de atención adecuada por parte del Estado al problema de la pobreza de estas personas. Por otra parte, a esto se unen las tendencias de la economía mundial hacia la precariedad y flexibilización del empleo, cuestión que muchas veces se ve expresado en este tipo de relación laboral con el Estado, donde no existen ningún tipo de seguridad laboral ni social que ampare a los trabajadores.

Otro elemento que resalta en la actual gestión de los servicios antimaláricos es la participación de diferentes órganos institucionales y de la comunidad, en las actividades de prevención y control de la malaria. Esto se había debilitado en los últimos años. Sin embargo, la gente ha venido mostrando preocupación y ha dado signos de una toma de conciencia acerca del problema que representa esta endemia, aún cuando para algunos todavía la malaria no representa un motivo de preocupación. De acuerdo a los testimonios de los médicos, enfermeras, promotoras sociales y de los propios pacientes, un ejemplo de la participación comunitaria lo constituye la experiencia en el municipio Mariño, allí el personal de salud desarrolló estrategias destinadas a lograr la participación de la sus habitantes en las actividades de atención, prevención y control sanitario. Específicamente, a través de grupos voluntarios de la comunidad, se lograron ejecutar actividades de saneamiento ambien-

16 Esta referencia numérica corresponde a la codificación de la información. En este caso se está

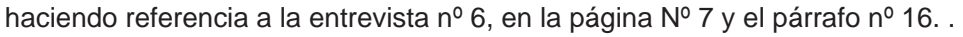

17 La población económicamente activa (PEA) en el municipio Mariño es de 13.025 personas pero sólo 5.737 de estas personas están ocupadas en la fuerza de trabajo (INE, 2001, cit. por Velásquez, 2005) 
tal, conformándose grupos de salud. De igual forma, se llevaron a cabo jornadas informativas casa por casa, sesiones educativas, charlas, incluso en compañía de los habitantes de las comunidades que integran este municipio, se realizaron jornadas de eliminación de criaderos que ellos mismos han aprendido a identificary a controlar por medio del uso de productos químicos (insecticidas).

Igualmente, la Gerencia de Saneamiento Ambiental y Contraloría Sanitaria ha tratado de involucrar a la mayor cantidad de organismos públicos, con el fin de fortalecer la idea de "intersectorialidad $^{\text {"18 }}$, es decir se ha tratado de desarrollar la capacidad cooperativa y/o de sinergia de los actores sociales. Se puede citar como ejemplo, el trabajo con el personal docente adscrito a los diferentes centros educativos que prestan servicio en las áreas de riesgo endémico y los intentos por involucrar a los integrantes del poder público local.

En relación a la intersectorialidad, un funcionario que labora a nivel directivo, manifestó lo siguiente:

"La integración fue un elemento fundamental, el acercamiento de diversos actores institucionales como las Alcaldías, las Juntas de Vecinos, MINFRA, Ministerio del Ambiente, Municipio Sanitario, Malariología o Gerencia de Saneamiento Ambiental y Contraloría Sanitaria, en- tre otros, han sido claves en el logro del éxito alcanzado". (17-04-09) (Médico).

\subsection{Opiniones respecto a la reestructuración}

La motivación del personal también ha tenido cabida dentro de la estrategia gerencial actual. Los reconocimientos han permitido la existencia de un clima laboral positivo ya que el personal ha mostrado actitudes favorables para el crecimiento y desarrollo de la organización. Además de la motivación, en las expresiones del personal se detecta el sentido de pertenencia, la solidaridad entre los compañeros de trabajo, la responsabilidad, el compromiso y el respeto a la institución y al mismo trabajo.

El personal que labora en las Ilamadas Demarcaciones y los médicos e inspectores sanitarios lo expresaron con estas palabras:

"Lo mejor de mi trabajo a mi criterio, trabajar con salud, yo estoy ayudando al campesinado, a la gente olvidada, porque nosotros nos metemos en los más mínimos rincones del estado Sucre y yo siento que yo estoy aportando algo de mi vida para mejorar el sistema de vida de la comunidad". (02-19-08) (Inspector Sanitario).

“(...) sinceramente, moralmente estoy dado para este trabajo (...) y el desempeño con las comunidades que es un

18 Intersectorialidad es una de las frases favoritas del equipo que trabaja en la prevención y control de la malaria en el estado sucre para referirse a la necesidad de incorporar a las diferentes instituciones y sectores de la vida social al trabajo de lucha antimalarica. Este mismo elemento de intersectorialidad, forma parte de uno de los principios fundamentales que expresa la Constitución de la República Bolivariana Venezolana para alcanzar el desarrollo social y económico del país. 
día a día (...) eso es lo mejor, eso es lo más bonito (...) uno se vuelve uno más de la comunidad, eso es experiencia”. (03-12- 03) (Inspector Sanitario)

"a nosotros nos enseñan a ser muy clínicos, pero no nos enseñan el abordaje de la comunidad, entonces nosotros hemos tenido que ir aprendiendo"(...). (1802- 05) (Médico).

"(...) sigo insistiendo en que nosotros somos héroes (...) asumimos con cariño y estamos empalagados de paludismo" (...). (01-07-09) (Médico).

“(...) tenemos grandes, grandes limitaciones pero muchas ganas de trabajary siempre me enorgullezco de nuestro estado Sucre y siempre digo somos sinvergüenza, no nos vamos, pero malaria es un vicio señores y aquí nadie se va, seguimos aquí de sin vergüenza" (0418-02) (Epidemiólogo).

Estas opiniones expresan la mística que ha desarrollado el personal de salud. La motivación desempeña un papel importante en la medida que influye en la productividad de la gente sobre todo si se toma en cuenta que este equipo de trabajo en su mayoría realiza su labor en condiciones adversas, ya que las comunidades presentan múltiples factores de riesgos cuyo control a veces escapa de la acción de este personal.

Otro aspecto importante es la formación del personal, el cual lógicamente necesita estar actualizado en el abordaje de los múltiples factores que se relacionan con este tipo de endemia. En tal sentido, se involucra al personal en charlas, talleres, seminarios para que nutran sus conocimientos y enfrenten adecuadamente las exigencias que diariamente se le presentan. En las gestiones pasadas se había descuidado la formación del personal, aún cuando en la gestión del Dr. Gabaldón siempre se insistió en la importancia de este elemento. La investigación también ha sido asumida como prioridad por parte del equipo gerencial no sólo a nivel de los factores físico-ambientales, biológicos, que puedan estar condicionando la presencia de la enfermedad, sino de los aspectos epidemiológicos, entomológicos que están asociados a ella e incluso de los aspectos socio-culturales $^{19}$.

Anteriormente, las actividades gerenciales estaban centradas en el control antivectorial, no obstante en la actualidad el personal de salud se mueve en torno a la idea de un manejo integral de la malaria, articulando actividades preventivas de educación para la salud, con investigaciones epidemiológicas, actividades de detección, diagnóstico, tratamiento oportuno e inmediato, vigilancia epidemiológica y actividades de control de vectores a nivel adulto y larvario.

19 La investigación que desarrolla el Grupo SOPHIS, con apoyo del FONACIT y de la Universidad de Oriente -y que cuenta con todo el respaldo del personal de salud que labora en la lucha antimalárica- es uno de esos intentos por investigar otros factores asociados a la prevalencia e incidencia de la enfermedad. En este caso, los factores socio-culturales y psico-sociológicos asociados a la emergencia y reemergencia de la Malaria. 
Dentro de los cambios gerenciales resaltan también las reuniones semanales que promueve la Gerencia en referencia. Estas reuniones vienen a conformar el espacio que agrupa a todos los funcionarios que tienen la responsabilidad de realizar el trabajo de atención, prevención y control de la malaria en el estado Sucre. La reunión "técnico-administrativa" como suele llamarse, cumple varias funciones: en ellas se da a conocer el reporte semanal acerca del comportamiento de la enfermedad, en los respectivos municipios sanitarios, se da a conocer verbalmente, se discute y se entrega por escrito la llamada "casuística" a nivel regional para cada "semana epidemiológica", también se discuten estrategias de acción para aquellas áreas críticas o necesitadas de un eventual reforzamiento de la actividad antimalárica y se diseñan y evalúan planes de trabajo en función de la situación epidemiológica presente en la región.

De igual manera, esta coordinación de esfuerzos se ha intentado llevar a un plano más amplio mediante la realización de reuniones a nivel inter-estadal, es decir con todos aquellos estados del país que tienen problema de transmisión malárica. Esto tiene como fin fortalecer el trabajo en red ya que se considera que las zonas de riesgos en otras entidades federales no deben permanecer como islas sino que debe darse el intercambio permanente para actuar en forma coordinada y así tener mayor efectividad en el objetivo común de detener el avance de esta endemia a nivel nacional.

Otro elemento clave de esta experiencia es que se han tratado de superar las limitaciones del proceso de descen- tralización a nivel regional. Esto se expresa en el hecho de que aún cuando muchos de los programas y políticas de salud continúan siendo elaboradas a nivel central, el equipo gerencial ha dado instrucciones a su personal para que adapten esos programas y propuestas a las realidades de cada municipio y de cada localidad, porque como ellos mismos dicen cada realidad es diferente y presentan condiciones epidemiológicas y culturales distintas, por lo tanto no se pueden aplicar las estrategias en forma homogénea.

"(...) la idiosincrasia de cada pueblo es diferente a otro, tú no puedes tratar una comunidad igualita a la otra, tienes que adaptarte a las comunidades (...)". (0907-01) (Médico).

En tal sentido, a pesar de que los lineamientos de políticas antimálaricas continúan como en el pasado, bajando del nivel central; mediatizando así el proceso de descentralización, el equipo que integra la Gerencia de Saneamiento Ambiental y Contraloría Sanitaria, ubicada en la ciudad de Carúpano, estado Sucre, está marcando una pauta ya que asume estos lineamientos como directrices generales y entienden que deben ser ajustados al contexto de cada localidad dependiendo de sus características epidemiológicas, ambientales, socioeconómicas y socio-culturales. De ahí que hay una planificación que aunque está orientada por las pautas que dicta el nivel central intenta ser coherente con la realidad que ellos tienen desde el punto de vista institucional, financiero, de la evolución de la enfermedad y de las respuestas que ofrecen otros actores sociales e instituciones (miembros de las comunidades 
afectadas, gobierno municipal, centros educativos, entre otros).

En otro orden de ideas, como parte del proceso generado por esta reestructuración de lo que anteriormente se conocía como Malariología, tenemos un cambio de racionalidad en relación al combate a la malaria en el estado Sucre. Anteriormente, predominaba una racionalidad netamente curativa, fundamentada en acciones de control anti-vectorial; sin embargo, a partir de los cambios antes citados, predomina un abordaje de la enfermedad desde una perspectiva integral, apoyada en la realización de actividades preventivas. De allí la importancia de la educación de las comunidades, la evaluación constante y oportuna y la investigación, cuestiones que permiten tomar decisiones fundamentadas en las condiciones epidemiológicas, económicas, sociales, culturales y físico-ambientales de las comunidades ubicadas en zonas de riesgo. Este cambio se nota, entre otras cosas, en el incremento de las actividades de educación sanitaria, de promoción de la participación comunitaria y de control mediante la búsqueda pasiva y activa
El incremento de la búsqueda pasi$\mathrm{va}^{20}$, en relación con períodos anteriores donde predominaba la búsqueda acti$\mathrm{va}^{21}$, es parte de las innovaciones. Es decir, hoy día el paciente acude voluntariamente, bien sea al cazador o a los puestos asistenciales, en busca de la atención médica, esto demuestra que hay un cambio de actitud en la comunidad, aunque esto no está generalizado en todas las áreas afectadas ya que aún se presenta la pasividad del paciente o la renuencia al tratamiento. Sin embargo, el aumento de la búsqueda pasiva representa un elemento importante que debe ser reforzado. Obviamente, no todo el trabajo está realizado, pero la merma de la endemia es un hecho, tal como lo muestran los indicadores de malaria para el año 2003, con un registro de casos significativamente menor ( 4.046 casos) que lo sucedido en el año anterior (en el año 2002 se presentaron 13.473 casos).

Un rasgo común en las entrevistas es que el personal de salud reconoce los beneficios de la reestructuración y el nuevo papel de las autoridades sanitarias locales; aunque también se mantiene la crítica hacia la forma cómo se actúo desde

20 El aumento de la Búsqueda Pasiva es un indicador para el personal de salud del impacto positivo que pueden producir las actividades socio educativas y preventivas dirigidas a la población afectada por la malaria. Dichas actividades se propone educar a la población para que en base a la información pueda identificar síntomas y acudir inmediatamente al centro de salud más próximo para que se le haga el respectivo examen diagnóstico y se le suministre el tratamiento si resultara positivo a la presencia de la malaria. El personal de salud dice que el incremento de la "búsqueda pasiva" significa que la gente ante la menor fiebre piensa en malaria.

21 A diferencia de lo anterior, la "Búsqueda Activa" se produce cuando el agente de salud (pastillero, en las antiguas gestiones; y cazador o visitador actualmente) se dirige a las comunidades y recorre una a una las viviendas de su comunidad preguntando acerca de la existencia de algún síntoma relacionado con la malaria (fundamentalmente fiebre). 
los niveles superiores con respecto a la tendencia al centralizadora que aún pervive y que se expresó en el manejo de esa reestructuración. Consideran que no fue un proceso que se generó en forma participativa. Existe la idea de que la reestructuración se inició en forma compulsiva, sin mirar, preguntar o escuchar al organismo que sería objeto de la misma. Ellos tienen la impresión que tan sólo se le asignaron responsabilidades, nuevas directrices y normas de trabajo, pero aún no gozan de suficiente autonomía, especialmente en el aspecto financiero

Algunas opiniones ofrecidas por el personal de salud expresan los retos y desafíos que representa para ellos la reestructuración del servicio antimalárico:

La reestructuración se da por que había la inquietud de que Malariología sola no podía asumir la responsabilidad de las subidas y bajadas de los Índices Parasitarios, del canal endémico. Precisamente hace aproximadamente tres años (2001-2002) se inició el proceso de reestructuración, eso no es fácil, yo creo que para nosotros ha sido un dolor de cabeza, no quiere decir que estemos magnificando el problema, ni evadiendo responsabilidad, ha sido un dolor de cabeza entrar a la disciplina que desde el año 35 se vienen creando situaciones de dificultad para su control, y este a veces, ... pensemos que de la noche a la mañana se va a controlar, entendemos que cada quien según su rol hace sus exigencias no, pero de la reestructuración para acá pienso que los progresos han sido enormes, el municipio ha asumido la responsabilidad que se le entregó, con muchos esfuerzos, o sea ese paso fue brusco y a veces sin escuchar al mismo municipio, si me equivoco y tengo la valentía de decirlo y si me corrigen a nosotros no se nos reunió para escuchar cual es la forma de reestructuración, nique opina usted sino, ahí te va (...)". (10- 5- 8) (Médico).

"... me ha tocado estar en la vieja y en la nueva Malariología y recuerdo que en Maracay ese fue uno de nuestros grandes temores, cómo le vamos a transferir a los municipios sin que ellos estén preparados para eso, y nosotros como institución tampoco estamos preparados para entregar eso y no se nos escuchó".(10- 05- 09). (Epidemiólogo).

"Esa reestructuración un poco compulsiva y sin consultar a nivel municipal, eso es una muestra y así se hace con los otros programas, pareciera como que a nivel municipal no tuvieran capacidad para dar una opinión, una propuesta (...) sin embargo, nosotros la asumimos con cariño y estamos empalagados de paludismo por que nos han permitido no sólo hacer ejercicios estadísticos, ejercicio de la disciplina en función del paludismo y hemos tenido que estudiar y renovarnos y la insistencia de los que están cerca del programa nos ha estimulado, nos ha hecho crecer $y$ estamos haciendo esfuerzos también para estar al día". (12-08-02) (Médico).

Adicionalmente, estos testimonios reflejan que la experiencia de reestructuración y descentralización en estudio no está ajena a los inconvenientes que a nivel general se han presentado desde que se produjo la firma del Convenio de Transferencia de los organismos de salud en el estado Sucre, en el año 1995. En un trabajo anterior relacionado con la Organizaciones Comunitarias de Salud 
(OCS) ya se habían detectado algunos problemas que nuevamente se vuelven a corroborar $^{22}$. Allí se planteaba la no realización efectiva de la descentralización administrativa debido a la no transferencia oportuna de los recursos financieros necesarios para agilizar el proceso de modernización del servicio de salud. Estos problemas también se refieren a lo siguiente:

"no se trata sólo de un asunto meramente financiero: existe también cierto desconocimiento de los funcionarios que laboran en el área de salud acerca de lo que significa conceptual y operativamente el proceso de descentralización. De modo que estas personas muchas veces se encuentran involucrados en decisiones relativas a lo que desde afuera se ha calificado como "proceso de modernización administrativa', sin que previamente se les haya orientado en forma clara acerca de la misión y visión que fundamenta esos cambios y lo que se espera que ellos hagan para operativizarlos" (Gómez, 2000: 11).

En el caso en estudio, tampoco se pudo cumplir en forma previa con la preparación del recurso humano -tanto la comunidad como el personal de saludpara el desarrollo de las tareas o funciones que demanda la nueva gestión descentralizada del servicio de salud. Aunque en la experiencia analizada los fun- cionarios de salud lograron superar en forma acertada esta limitación, no sucede lo mismo con los demás actores comunitarios -grupos de salud, OCS, asociaciones de vecinos, pacientes y no pacientes y en general, los habitantes de la comunidad- Teóricamente, estos actores deben insertarse para darle un carácter participativo y de corresponsabilidad, al servicio de atención, prevención y control de la malaria pero en la práctica suele existir una brecha enorme entre lo normativo $y$ la realidad.

\section{Consideraciones finales}

La revisión de esta experiencia de gestión en la lucha contra la malaria, ha puesto de manifiesto tres escenarios fundamentales en los que se ha movido esta lucha en Venezuela y particularmente en el estado Sucre. El primero, se caracterizó por la pésima situación socio-económica y política existente en el país en la década de los treinta, que agudizó también el panorama higiénico-sanitario y epidemiológico nacional; sin embargo, dicho panorama en lo que se refiere a esta enfermedad, pudo posteriormente revertirse gracias a la estrategia gerencial implementada por el Dr. Arnoldo Gabaldón. En el segundo escenario, nos encontramos con un país que descuida las políticas socio-sanitarias, aplica medidas eco-

22 En relación a las OCS como organizaciones de participación comunitaria en el control y prevención de la salud, surgidas en el marco de las estrategias formuladas por FUNDASALUD para el período 1996-2000, también se detectaron una serie de problemas que se resumen en lo siguiente: desigual concentración geográfica; fomento de un tipo participación controlada desde el Estado, relación con las instancias de gobierno en algunos casos, sesgadas por el clientelismo y la dependencia político-partidista (Alves, 2001). 
nómicas que lejos de mejorar la situación social la agudizan aún más, permitiendo entre otras cosas, la emergencia y reemergencia de enfermedades infecto-contagiosas aparentemente erradicadas, tales como la malaria. En el tercer escenario, nos encontramos con una situación epidemiológica delicada y con tendencia al agravamiento. Esto dio lugar a un proceso de reestructuración de la institución encargada de prestar servicios antimaláricos y a la realización de un conjunto de cambios que no sólo permitieron el otorgamiento de responsabilidades y la emisión de respuestas oportunas, sino también -y es lo más importante- la disminución de la endemia en una parte del país.

En fin, los escenarios en los que se ha movido el control malárico en Venezuela revelan la importancia de implementar cambios y procesos innovadores a nivel de las políticas y estrategias gerenciales de las instituciones públicas, cualquiera sea su naturaleza, porque éstos permiten alcanzar resultados positivos que contribuyen al bienestar de la población beneficiaria, como sucedió en la experiencia estudiada.

En síntesis, la estrategia gerencial consistió básicamente en la reorganización de la antigua Malariología, fusionándola con los servicios asistenciales de salud, representados en los Municipios Sanitarios. Esta estrategia gerencial incorporó como parte del equipo antimalàrico, al personal de salud tradicionalmente ocupado en la atención de otras patologías, y a la figura de los cazadores de malaria, estas cuestiones fueron puntos clave en el éxito alcanzado en la disminución de la enfermedad. Los cazadores consti- tuyen parte importante del enlace entre la comunidad y la institución prestadora del servicio de salud. A través de este personal, la GSACS-Carúpano mantiene el monitoreo y una vigilancia epidemiológica permanente y activa en las comunidades. Sin embargo, es un personal cuyo status laboral debe ser redefinido, ya que la condición de personal voluntario o "colaborador" no se corresponde con la magnitud del trabajo antimálarico que llevan a cabo y menos con su situación socio-económica y socio-laboral. Da la impresión que en esta situación -de no mejoramiento de las condiciones laborales de los cazadores- está influyendo un poco la visión que algunos funcionarios de salud tienen acerca de lo que debe ser la participación de la comunidad en el campo de la salud. No estando clara la separación entre los conceptos de trabajo voluntario, voluntariado y personal que cumple una función o trabajo que es básico para el organismo de salud

Por otra parte, el proceso de reestructuración en referencia, permitió un avance significativo en el estilo de gerencia de la salud en Venezuela; fundamentado en la horizontalidad y en una orientación de abajo hacia arriba, lo cual ha permitido la formación de un equipo gerencial con un gran sentido de pertenencia y respeto por el trabajo. La comunicación abierta y constante entre los integrantes del equipo también constituye uno de los elementos claves que caracterizan la actual gestión de este servicio de salud en la región sucrense. Es una experiencia que aprovechando el escenario de la descentralización, debería ser multiplicada en otras áreas del país que presenten este u otro problema de salud. 
Finalmente, otra lectura de esta experiencia es que en el estado Sucre se están dando significativos avances en la idea de la salud-enfermedad, como un proceso. Ya no sólo se trata de una visión en términos de presencia o ausencia de enfermedades, donde se legitima el modelo curativo. Hay una tendencia a privilegiar la prevención de enfermedades y la promoción de la salud como valores fundamentales. Esto significa que se están dando pasos importantes que pueden significar un cambio de paradigma favorable a la implementación exitosa de las políticas de salud en Venezuela.

\section{Referencias Bibliográficas}

Alves, Julissa (2001). Operatividad de la descentralización del sector salud en la promoción de la participación organizada de las comunidades en el municipio Sucre, estado Sucre. Tesis de Grado. Universidad de Oriente. Cumaná.

Cumana Yaneira y Sucre Yuraima (2005). Organizaciones sociales y su participación en la gestión pública en el marco de la descentralización. Caso: Organizaciones comunitarias de salud ene. Estado Sucre. Tesis de Grado. Universidad de Oriente. Cumaná.

Gerencia de Saneamiento Ambiental y Contraloría Sanitaria (2002). Reportes epidemiológicos de la malaria en el estado Sucre. Carúpano, estado Sucre. Versión electrónica enviada al equipo de investigación.

Gómez, Irey (2000). Significado y alcance de la participación de la comunidad en la descentralización del servicio de salud. Revisión de experiencias. Ponencia presentada a la L Conven- ción de ASOVac. Universidad Simón Bolívar. Caracas. Venezuela.

Gutiérrez, Ana. (1998). Tiempos de guerra y paz. Arnoldo Gabaldón y la investigación sobre malaria en Venezuela 1936-1990. CENDES. Universidad Central de Venezuela. Caracas, Venezuela.

Gutiérrez, Ana (1999). El Conocimiento Traducido: El Programa de Control Antimalàrico Venezolano (1936 - 1945). En Cuadernos del CENDES, Año 16, No. 41. CENDES-UCV. Caracas. pp. 153- 172.

Grupo de Investigación e Innovaciones Sociales-SOPHIS- Proyecto UDO-FONACIT. Entrevistas realizadas al personal de salud de la GSACS-Carúpano, durante el período 20032004. La desgrabación y transcripción de este material fue realizado bajo la asesoría y responsabilidad del equipo investigador ubicado en la Universidad de Oriente. Cumaná-estado Sucre.

Kliksberg, Bernardo. (2003). Hacia una gerencia social eficiente: algunas cuestiones claves. Banco Interamericano de Desarrollo. s/i.

Kliksberg, Bernardo. (s/f. a). ¿Cómo formar gerentes sociales? Elementos para el diseño de estrategias. Material mimeografiado.

Molina, María y Morera, Nidia. (1999). La Gerencia de los Servicios Sociales. Buenos Aires, Editorial LUMEN/HVMANITAS.

Montes, Marcelino. (2001). ¿Hay alguna diferencia entre Paludismo y Malaria? En PROVINCIA, Cumaná, 14 de marzo de 2001, estado Sucre, Venezuela

Organización Mundial de la Salud y Organización Panamericana de la Salud.(OMS/OPS), (2000). Informe sobre la situación de los Programas de Malaria en las Américas. Disponi- 
ble en: http://www.paho.org . Revisión de 17 de febrero 2004.

Organización Panamericana de la Salud. (OPS), (2001). Situación de los Programas de Malaria en las Américas. En Boletín Epidemiológico, Año. 1, No. 22. Disponible en: http://www.paho.org . Revisión de 27 de enero de 2004.

Velásquez, Ysabel (2005). La malaria: una perspectiva desde las representaciones sociales. Casos Río Seco y Río Chiquito Arriba. Municipio Mariño. Estado Sucre. Tesis de Grado. Universidad de Oriente. Cumaná, estado Sucre (versión preliminar). 